\title{
A Novel Compact Stand-alone FTIR Microscope for the Analysis of Small Samples
}

Thomas J Tague Jr.

Bruker Optics, Inc., 19 Fortune Drive, Billerica, MA 01821

Fourier Transform Infrared (FTIR) microscopy is a well-established method for the analysis of samples that are too small or complex to be measured in a standard infrared spectrometer. With FTIR microscopy, it is possible to readily visualize the sample and determine the area(s) of interest. IR spectra are then easily collected with excellent spatial resolution, thereby revealing the chemical composition of the area of interest. Since it is possible to analyze a large variety of different samples like fibers, paint chips, contaminations or composite materials (e.g. laminates, tablets) there are countless possible applications. So far most of the commercially available FTIR microscopes are rather bulky and need to be attached to a FTIR spectrometer to received modulated infrared light. Furthermore, they are quite complex to use and require a high level of skill. The new LUMOS FTIR microscope is a new fully automated stand-alone solution that is very easy-to-use and requires very little lab space. The OPUS software is a comprehensive microanalysis platform that incorporates a novel wizard that makes sample visualization, data collection, and resulting data analysis quick and easy. For the first time, no experience of training is necessary to perform diffraction limited infrared microanalysis.

The LUMOS FTIR microscope, shown in Figure 1, is an all-in-one solution with an integrated FTIR spectrometer, a high degree of motorization and a dedicated user interface. It also utilizes state-of-the-art optics for optimal sample visualization and infrared data collection. The Lumos uses a new approach for sample visualization utilizing an $8 \mathrm{x}$ Schwartzchild reflecting objective with a variable numerical aperture (NA). Optimal sample visualization is performed with a NA of 0.4 and optimal data collection in ATR, transmission, and reflection modes is accomplished with a NA of 0.6. The innovative highly accurate piezo drive attenuated total reflectance (ATR) acquisition mode performs the complete measurement procedure fully automated including background and sample measurements. To provide perfect contact of the ATR crystal with samples ranging from soft to very hard, contact pressure is monitored precisely and is software selectable.

Paint chip samples are frequently too brittle for thin sectioning so the measurement was conducted in ATR mode. In this example, a polished sample removed from a painting is analyzed by drawing the area of interest and utilizing automated knife edge apertures. The data was collected at 32 scans per measurement point with a spectral resolution of $4 \mathrm{~cm}-1$. During the infrared analysis, the sample was brought into contact with the tip of the Germanium (Ge) ATR crystal on the predefined measurement positions. Figure 2 shows the lead sulfate chemical image from the middle white layer with the corresponding measurement grid shown by the red boxes. Simply clicking on the visible or chemical image and selecting search spectrum provides a rapid identification using the extensive infrared spectrum database. All layers were readily identified by their respective spectra. 
Chemical imaging using IR microscopy is an established technique for the investigation of samples with complex chemical structures. The fourth example is a small piece of onion tissue which was mapped in transmission mode. The chemical image shown in Figure 3 consists of $58 \times 47$ measurement points and was recorded with an acquisition time of 1.5 seconds/position with a resolution of $15 \times 15 \mu \mathrm{m}$ and a spectral resolution of $8 \mathrm{~cm}-1$. The chemical image is the integration result of the lipid band at $1740 \mathrm{~cm}-1$ superimposed with the visual image. Both images match perfectly and the single cells are clearly visible. The color coding of the chemical image shows the distribution of the lipid band and therefore the distribution of the lipids in the cells. As expected, the highest lipid concentration can be found in the cell walls since they contain a lipid bilayer.

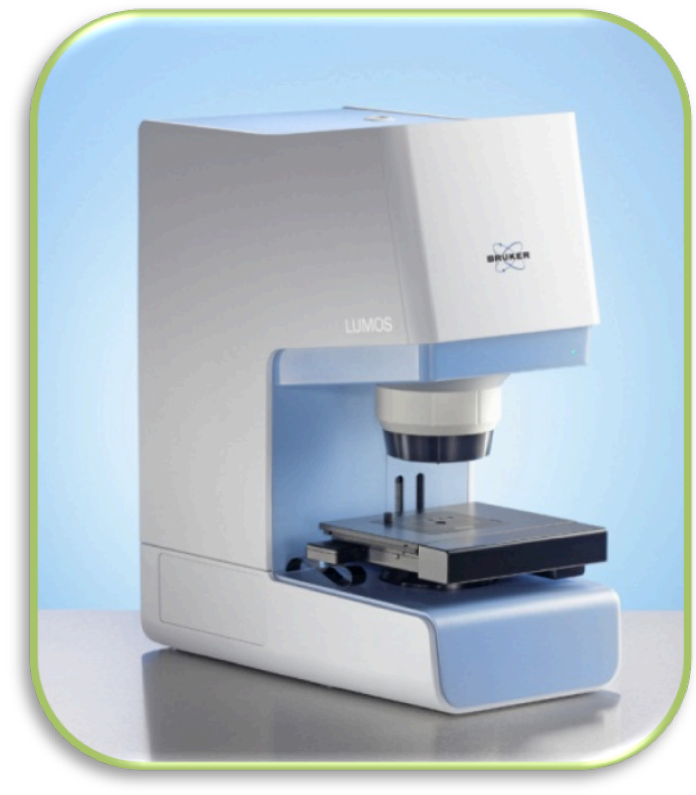

Figure 1 - The stand-alone Lumos infrared microscope.

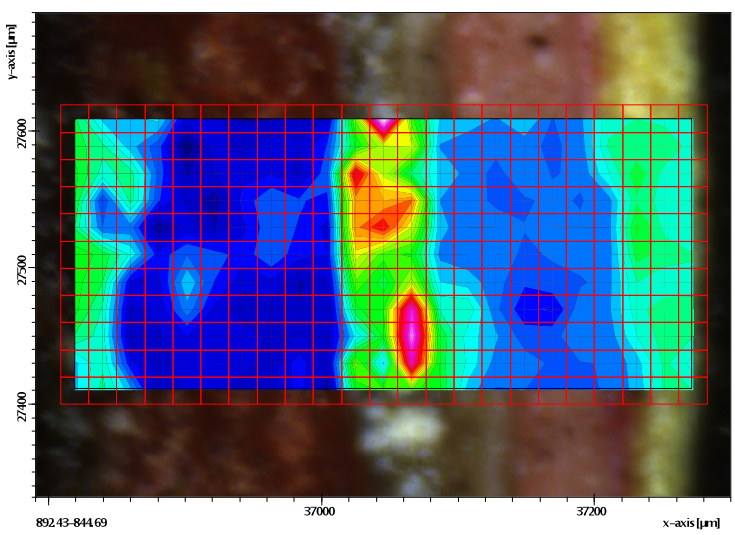

Figure 2 - Polarized light image of a cross section removed from an old painting with the lead white chemical image superimposed.

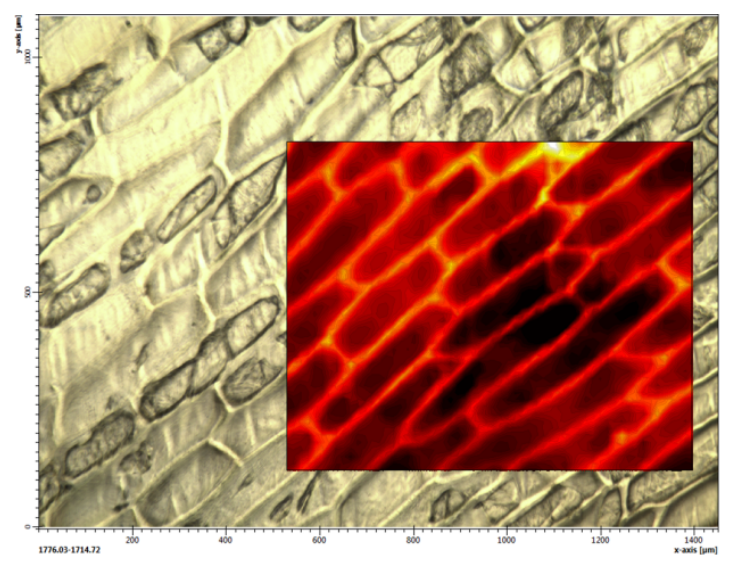

Figure 3 - Brightfield image of an onion tissue with the overlaid lipid chemical image. 